Report 1999-050

Admission Policies

for a Two Class Loss System

E. Lerzan Örmeci, Apostolos Burnetas and Jan van der Wal

ISSN 1389-2355 


\title{
Admission Policies for a Two Class Loss System
}

\author{
E. Lerzan Örmeci, Apostolos Burnetas and Jan van der Wal
}

January 24, 2000

\begin{abstract}
We consider the problem of dynamic admission control in a Markovian loss queueing system with two classes of customers with different service rates and revenues. We establish the existence of optimal acceptance thresholds for both customer classes. We also show that under certain conditions there exists a preferred class. These results are valid under a Markov arrival process.
\end{abstract}

\section{Introduction}

Dynamic admission control strategies are of increasing importance as revenue management tools in service and manufacturing systems. In telecommunications and in particular in telephone service and support applications, such strategies are commonly used in order to increase flexibility in the allocation of resources among different customer types.

In a queueing context, a static admission rule specifies a priori whether each customer class is admitted for service, based on the revenue that customers of this class generate and their expected service requirements. This specification is made independently of the system state and is equivalent to determining whether one customer class is preferred over the other. On the other hand, a dynamic admission policy offers increased flexibility because it makes the decision of admitting an arriving customer contingent on the current level of congestion upon arrival, in addition to the customer's class. A dynamic admission policy is equivalent to a resource idling rule. Indeed, when a customer is not admitted for service, it is effectively decided to keep one or more servers idle in anticipation of future admission of more profitable customers who would otherwise be lost.

In this paper we consider the problem of dynamic admission control in a two class loss Markovian queueing system with different service rates for the two customer classes. We prove that an optimal admission policy can be generally described as follows. An arriving customer is admitted to the system if and only if the number of available servers exceeds a certain threshold, which depends on the number of customers of both classes already being served. In addition we develop a set of sufficient conditions which ensure that a customer class is "preferred", in the sense that its customers are always admitted if there are free servers, regardless of the system congestion level. Finally, we show via a counterexample that even when a preferred class exists, it is not necessarily characterized by the popular $c \mu$ rule in the stochastic scheduling literature.

The model we study is a loss system with $c$ identical parallel servers, no waiting room and two classes of customers. Class- $i$ customers arrive according to a Poisson process with 
rate $\lambda_{i}$ and demand an exponential service time with mean $1 / \mu_{i}$. If a class- $i$ customer is admitted, a reward of $r_{i}>0$ is gained after his (her) service is finished. Our objective is to find dynamic admission policies that maximize the total expected discounted revenue over a finite or infinite horizon as well as the long-run average revenue.

A special case of the model described above, with $\mu_{1}=\mu_{2}$ and $r_{1}>r_{2}$, is studied in Miller (1971), where the optimal policy is shown to accept class-1 customers whenever there is an idle server and to admit class- 2 customers if and only if the total number of customers in the system is below a certain threshold. Thus, the optimal policy in Miller's work is a threshold type policy with a preferred class. Lippman \& Ross (1971) analyze the optimal admission rule for a system with one server and no waiting room which receives offers from customers according to a joint service time and reward probability distribution (this model is usually referred to as the streetwalker's dilemma). Whenever the service times are exponential and rewards are fixed, this is also a special case of our model with $c=1$. In both of these studies, there exists a preferred class which is determined by the $r \mu$ rule, the equivalent of the $c \mu$ rule in these systems. Recall that the $c \mu$ rule gives priority to the class with the highest average cost rate, $c_{i} \mu_{i}$, where $c_{i}$ is the holding cost and $\mu_{i}$ is the service rate of class- $i$ customers, so this class is preferred over the others. With the objective of maximizing revenue, this rule translates to the $r \mu$ rule, since the quantity equivalent to $c_{i} \mu_{i}$ is the average profit rate of class- $i$ customers, $r_{i} \mu_{i}$. The results of these two studies suggest that in our model, too, the $r \mu$ rule may determine the preferred class. However, this is not the case in general, as we see with a counterexample in Section 5 . It should be noted that the term "preferred" is used here to denote a class whose customers are always admitted to the system when there is at least one free server, so that there can be no preferred class at all or there can be more than one preferred class. This terminology reflects the preferential treatment that a class may enjoy in the sense that it is never rejected unless the system is full. It is therefore a global state independent property. With a different service rate and reward for each class, it is not clear whether a preferred class exists and, if it does, how to determine it. One additional difficulty stems from the fact that availability of jobs is also of concern since we have a loss system. Thus, the arrival rates also affect the determination of preferred class(es).

We can see that each class has its own advantage and disadvantage for being a preferred class. The strength of class- 1 customers is the steady returns they bring to the system because of the longer service times: Consider a firm which has two kinds of jobs, one of which brings $\$ 1,000$ profit each month for 12 months and the other with a profit of $\$ 1,200$ per month for only 3 months. The possibility that the firm will have no job after 3 months works in favor of the longer duration job. We show that under certain conditions, a preferred class exists, and under a stronger set of conditions, the preferred class is determined by the $r \mu$ rule.

We prove the existence of an optimal admission policy that is characterized by acceptance thresholds for both classes. Specifically, for class-2 customers, there exist thresholds such that the optimal policy accepts class- 2 customers only if the number of class- 1 customers in the system is less than a specified threshold, and rejects otherwise. The thresholds depend on the number of class- 2 customers already in the system. The admission policy for class- 1 customers can be described similarly. We also show monotonicity of thresholds under very restrictive conditions. 
We finally establish that the above results are still valid under a Markov arrival process (MAP), which is shown to approximate any independent arrival process arbitrarily closely in Asmussen \& Koole (1993).

There has been an increasing interest in multiclass loss networks due to the growth of telecommunications systems. Admission control is a main focus of research on loss networks (see Chapter 4 of Ross (1995) for a comprehensive review). The work in this area is usually directed towards analyzing certain classes of policies (e.g., coordinate convex policies, trunk reservation policies), although there are studies that address the issue of the optimal policy directly such as Miller (1971) and Lippman \& Ross (1971). More recently, Ku \& Jordan (1997) consider two stations in tandem, each with no waiting room and parallel servers. Carrizosa, Conde \& Munoz-Marquez (1998) present an optimal static control policy for acceptance/rejection of $k$ classes in an $M / G / c / c$ queue. In all these studies, there exists a preferred class which is determined by a variation of the $c \mu$ rule. The $c \mu$ rule has been shown to be optimal in various queueing systems, see e.g., Baras, Dorsey \& Makowski (1985), Buyukkoc, Varaiya \& Walrand (1985), Varaiya, Walrand \& Buyukkoc (1985).

This paper is organized as follows: In the next section, we develop a Markov Decision Process (MDP) model for the system described above. In Section 3 we prove the existence of an optimal threshold policy. In Section 4 we present sets of sufficient conditions for each class to be preferred. Section 5 discusses the issues regarding to preferred classes, and includes a counterexample to the $r \mu$ rule. In Section 6 we show that under certain conditions the optimal acceptance thresholds have intuitive monotonicity properties. Section 7 discusses extensions.

\section{Markov Decision Model of the System}

\subsection{Discrete time model of the system}

The original process of the system described above evolves in continuous time. All the interarrival times as well as the service times are exponential. Furthermore, we interpret discounting as exponential failures, i.e., the system closes down in an exponentially distributed time with rate $\beta$ (for the equivalence of the process with discounting and the process without discounting but with an exponential deadline, see e.g., Walrand (1988)). Thus, the state of the system can be described by $x=\left(x_{1}, x_{2}\right)$, where $x_{i}$ is the number of class- $i$ customers in the system. If the system is in state $x$, an arrival of class $j$ will occur with a rate of $\lambda_{j}$, a class- $i$ customer will depart from the system with a rate of $x_{i} \mu_{i}$ and the system will close down with no further return with rate $\beta$. We can build a discrete time equivalent of this system by using uniformization: First, we assume, without loss of generality, $\mu_{1}<\mu_{2}$; note that the case $\mu_{1}=\mu_{2}$ was covered by Miller (1971). Then, the maximum possible rate out of any state, say $A$, is $\lambda_{1}+\lambda_{2}+c \mu_{2}+\beta$. Now, if the system is in state $x$, a potential transition will occur with rate $A$, and the actual transition will be a class- $j$ arrival with probability $\lambda_{j} / A$, a class- $i$ service completion with probability $x_{i} \mu_{i} / A$, a "fictitious" service completion, which does not change the state of the system, with probability $\left(c \mu_{2}-x_{1} \mu_{1}-x_{2} \mu_{2}\right) / A$, and finally the system will close down with probability $\beta / A$. We observe the state of the system at each instant of a potential transition, so in every exponentially distributed time with rate $A$, and the above probabilities give the actual transition of the system. Thus, the discrete times correspond to the number 
of remaining potential transitions. Now, we can use normalization as well, so that we can assume, using the appropriate time scale, $A=1$. Then the system will be observed in exponentially distributed intervals with mean 1 , and, as described before, there will be an arrival with probability $\lambda_{1}+\lambda_{2}$ and a potential service completion with probability $c \mu_{2}$.

The assumption $\mu_{1}<\mu_{2}$ implies that class- 1 customers are "slow" customers. We use this assumption quite often to couple the service times of class- 1 and class- 2 customers. If we want to couple service times of a certain class- 1 customer, say $d_{1}$, and a class- 2 customer, say $d_{2}$, we let $\xi$ be a uniformly distributed random variable in $(0,1)$, and we generate the service times of $d_{1}$ and $d_{2}$ using the same $\xi$, so customer $d_{2}$ leaves earlier than customer $d_{1}$ leaves with probability 1 . In terms of discrete time, this translates to the following: Both customers leave the system with probability $\mu_{1}$, and a class- 2 customer departs from the system with probability $\mu_{2}-\mu_{1}$ leaving the coupled class- 1 customer in the system. Thus, coupling never allows a coupled class- 1 customer to leave the system while the coupled class- 2 customer is still there.

We define the state of the system in the discrete time MDP including the last event occurred: If there is a potential service completion, we denote the system state by $x=$ $\left(x_{1}, x_{2}\right)$, where $x_{i}$ is the number of class- $i$ customers. If there is a class- $j$ arrival, then the state is $(x ; j)=\left(x_{1}, x_{2} ; j\right)$ so that there are $x_{i}$ class- $i$ customers in the system and a class- $j$ customer has just arrived. Distinguishing the last event occurred in the state of the system is quite artificial, but it reflects the consequences of actions more clearly, as we see from the optimality equations given in the next subsection. Note that we always have $x_{1}+x_{2} \leq c$ and the actions are defined only for the states corresponding to an arrival.

\subsection{Markov decision model for finite horizon}

Let $u_{n}(x)\left(v_{n}(x ; j)\right)$ be the maximal expected $\beta$-discounted reward for the system starting in state $x((x ; j))$ when $n$ observation points remain in the horizon. Let $\mathcal{S}$ be the set on which $u_{n}$ 's are defined, i.e., $\mathcal{S}=\left\{x: x_{1}+x_{2} \leq c\right\}$. We define $a_{n}(x ; j)$ as the optimal action in state $(x ; j)$ when there are $n$ more transitions. $a_{n}(x ; j)$ is set to 1 if it is optimal to accept the arriving customer of class $j$ and to 0 otherwise. Now we present the optimality equations. Setting $e_{j}$ as the vector which has a 1 at the $j$ th coordinate, and 0 elsewhere, we have the following for $x_{1}+x_{2}<c$ :

$$
\begin{aligned}
v_{n}(x ; j)= & \max \left\{u_{n}\left(x+e_{j}\right), u_{n}(x)\right\} \quad \text { for } j=1,2 \\
u_{n+1}(x)= & x_{1} \mu_{1} r_{1}+x_{2} \mu_{2} r_{2}+\lambda_{1} v_{n}(x ; 1)+\lambda_{2} v_{n}(x ; 2)+ \\
& x_{1} \mu_{1} u_{n}\left(x-e_{1}\right)+x_{2} \mu_{2} u_{n}\left(x-e_{2}\right)+ \\
& \left(c \mu_{2}-x_{1} \mu_{1}-x_{2} \mu_{2}\right) u_{n}(x)
\end{aligned}
$$

and for $x_{1}+x_{2}=c, a_{n}(x ; 1)=a_{n}(x ; 2)=0$ and so

$$
v_{n}(x ; 1)=v_{n}(x ; 2)=u_{n}(x)
$$

where $u_{n}\left(-1, x_{2}\right)=u_{n}\left(0, x_{2}\right)$ and $u_{n}\left(x_{1},-1\right)=u_{n}\left(x_{1}, 0\right)$. If the last event occurred is a class- $j$ arrival, which happens with probability $\lambda_{j}$, (s)he is either accepted so that the system moves to the state $x+e_{j}$, or rejected, which keeps the system in the same state $x$. If a class- $i$ customer finishes his service, with probability $x_{i} \mu_{i}$, the system state changes to 
$x-e_{i}$ with a reward of $r_{i}$. The "fictitious" service completions, which occur with probability $c \mu_{2}-x_{1} \mu_{1}-x_{2} \mu_{2}$, affect neither the state nor the total reward of the system. Finally, if the system closes down, with probability $\beta$, the system receives no more reward.

\subsection{Infinite horizon models}

We prove all our results for the objective of maximizing total expected $\beta$-discounted reward for a finite number of transitions, $n$, including the "fictitious" transitions due to the "fictitious" service completions. Thus, "finite" horizon problems are pseudo finite problems. They provide the powerful tool of induction to prove our results for all $n$, which allows us to consider the infinite horizon problems: All the results proven for finite $n$ are true for the limit $n \rightarrow \infty$, so the corresponding conclusions are valid when total expected $\beta$-discounted reward over an infinite horizon is maximized. Moreover, since the state space and the action space in each state are finite and the results hold for all $\beta$, including $\beta=0$, we have the same conclusions for maximizing the long-run average reward. Here, we note that for the results regarding to the preferred class, we specify the initial value function $u_{0}$ in such a way that the rewards of customers, who are still in the system at $n=0$, are collected even if their services have not been finished. Of course, this makes no difference in the optimal policy for infinite horizon problems.

We define $v(x ; j)(u(x))$ as the maximal expected $\beta$-discounted reward for the system starting in state $(x ; j)(x)$ over an infinite horizon. Thus, for $\beta>0$, we have:

$$
\begin{aligned}
v(x ; j) & =\lim _{n \rightarrow \infty} v_{n}(x ; j) \\
u(x) & =\lim _{n \rightarrow \infty} u_{n}(x)
\end{aligned}
$$

$a(x ; j)$ is the corresponding action in state $(x ; j)$ so that $a(x ; j)=1$ if the customer is accepted and $a(x ; j)=0$ otherwise. For $\beta=0, u(x) \rightarrow \infty$, so we need to consider the relative value functions and the gain in the usual MDP formulation.

\subsection{Effect of an additional customer}

We choose to serve a customer, if both rejecting and accepting him (her) is optimal. Then, from equation (1), it is easy to see that

$$
a_{n}(x ; j)=1 \Longleftrightarrow 0 \leq u_{n}\left(x+e_{j}\right)-u_{n}(x) .
$$

We can interpret the difference $u_{n}\left(x+e_{j}\right)-u_{n}(x)$ as the benefit of the system due to an additional class- $j$ customer in state $x$ when there are $n$ more transitions. Then, the optimal policy accepts a class- $j$ customer only if the benefit (s)he brings to the system is positive. These differences play a crucial role in determining preferred class(es). Therefore, we define $D_{n}(i j)(x)$ as the difference in the total expected discounted rewards between system 1 and system 2 if system 1 starts in state $x$ 'plus' one class- $i$ job and system 2 starts in $x$ plus a class- $j$ job, where $j=0$ means that system 2 is in state $x$, i.e., there is no additional customer. We, occasionally, drop the arguments $x$ and $n$ later on, when there is no danger of confusion in the reference. The four $D_{n}(i j)$ functions of interest are $D_{n}(10), D_{n}(20), D_{n}(12)$ and $D_{n}(21)$. It is easy to see that $D_{n}(10)(x)=u_{n}\left(x+e_{1}\right)-u_{n}(x)$, $D_{n}(20)(x)=u_{n}\left(x+e_{2}\right)-u_{n}(x)$ and $D_{n}(12)(x)=-D_{n}(21)(x)=u_{n}\left(x+e_{1}\right)-u_{n}\left(x+e_{2}\right)$. 
Hence, the difference $D_{n}(i 0)(x)$ corresponds to the net benefit of the system due to an additional class- $i$ customer in state $x$ when there are $n$ more transitions, whereas $D_{n}(12)(x)$ is the net benefit of the system when a class- 1 customer already in the system is changed to a class- 2 customer in state $x+e_{1}$.

Now, we can identify a preferred class in terms of $D_{n}(i j)$ 's. First, we note that $a_{n}(x ; j)=$ 1 if and only if $0 \leq D_{n}(j 0)(x)$. Then, if $D_{n}(j 0)(x) \geq 0$ for all $x \in \mathcal{S}$, class- $j$ customers are preferred; since if the net benefit of accepting a class- $j$ customer is non-negative for all states, then class $j$ is a preferred class.

\subsection{A remark on rewards}

In this model, we have considered only the rewards collected at the end of service. Rejection costs, say $b_{i}$, which are incurred at the time of the arrival of a rejected customer can be incorporated in the model by redefining the reward $r_{i}$ as $r_{i}+\frac{\mu_{i}+\beta}{\mu_{i}} b_{i}$ due to the discounting. For a more general system with both rejection costs, $b_{i}$, and rewards, $r_{i}$, one can refer to the thesis Örmeci (1998), where all the equivalent results of this paper are stated with a more complicated notation, although the methods of proofs with or without rejection costs are the same.

The present value of the reward brought by a class- $i$ customer is $\frac{r_{i} \mu_{i}}{\mu_{i}+\beta}$ due to the discounting. We refer to this quantity as the immediate reward of a class- $i$ customer and denote it by $R_{i}$. Thus, $r_{i}$ is the value of the reward in the end of service, whereas $R_{i}$ is its value in the beginning of the service. Another quantity of interest is the average reward of a class- $i$ customer, $r_{i} \mu_{i}$.

\section{Existence of an optimal threshold policy}

In this section we show that there exists an optimal policy which can be determined by optimal thresholds. For this, we prove that the value functions, $u_{n}$ 's, satisfy the following monotonicity for all $n$ whenever $u_{0}$ does.

Lemma 1 For all $x$ with $x_{1}+x_{2}+2 \leq c$ (or equivalently for all $x+e_{1}+e_{2} \in \mathcal{S}$ ):

$$
u_{n}(x)-u_{n}\left(x+e_{2}\right)-u_{n}\left(x+e_{1}\right)+u_{n}\left(x+e_{1}+e_{2}\right) \leq 0 \quad \forall n \geq 1,
$$

whenever the inequality is true for $n=0$.

Proof. Assume that $u_{0}$ satisfies the above inequality. Note that many functions satisfy this inequality including $u_{0}(x)=0$ for all $x$ and $u_{0}$ given by equation (3). We prove the statement by induction on the number of remaining transitions, so assume:

$$
u_{n}(x)-u_{n}\left(x+e_{2}\right)-u_{n}\left(x+e_{1}\right)+u_{n}\left(x+e_{1}+e_{2}\right) \leq 0 \quad \forall x+e_{1}+e_{2} \in \mathcal{S}
$$

We first show that the $v_{n}(. ; j)$ 's also satisfy this monotonicity, so let $\delta_{j}$ be such that:

$$
\delta_{n}(j)=v_{n}(x ; j)-v_{n}\left(x+e_{2} ; j\right)-v_{n}\left(x+e_{1} ; j\right)+v_{n}\left(x+e_{1}+e_{2} ; j\right) .
$$

We consider the four possible cases due to actions $a_{n}(x ; 1)$ and $a_{n}\left(x+e_{1}+e_{2} ; 1\right)$ upon class-1 arrivals: 
Case I: $a_{n}(x ; 1)=a_{n}\left(x+e_{1}+e_{2} ; 1\right)=0$

$$
\delta_{n}(1) \leq u_{n}(x)-u_{n}\left(x+e_{2}\right)-u_{n}\left(x+e_{1}\right)+u_{n}\left(x+e_{1}+e_{2}\right) \leq 0
$$

where the first inequality follows from the case assumptions and the optimality of the $v_{n}$ 's and the second one from the induction hypothesis.

Case II: $a_{n}(x ; 1)=1$ and $a_{n}\left(x+e_{1}+e_{2} ; 1\right)=0$

$$
\delta_{n}(1) \leq u_{n}\left(x+e_{1}\right)-u_{n}\left(x+e_{2}+e_{1}\right)-u_{n}\left(x+e_{1}\right)+u_{n}\left(x+e_{1}+e_{2}\right)=0
$$

where the inequality follows from the case assumptions and the optimality of the $v_{n}$ 's.

Case III: $a_{n}(x ; 1)=0$ and $a_{n}\left(x+e_{1}+e_{2} ; 1\right)=1$

$$
\begin{aligned}
\delta_{n}(1) \leq & u_{n}(x)-u_{n}\left(x+e_{2}\right)-u_{n}\left(x+2 e_{1}\right)+u_{n}\left(x+2 e_{1}+e_{2}\right) \\
= & u_{n}(x)-u_{n}\left(x+e_{2}\right)-u_{n}\left(x+e_{1}\right)+u_{n}\left(x+e_{1}+e_{2}\right) \\
& +u_{n}\left(x+e_{1}\right)-u_{n}\left(x+e_{1}+e_{2}\right)-u_{n}\left(x+2 e_{1}\right)+u_{n}\left(x+2 e_{1}+e_{2}\right) \\
\leq & 0
\end{aligned}
$$

where the first inequality is true due to the case assumptions and the optimality of the $v_{n}$ 's and the second one due to the induction hypothesis.

Case IV: $a_{n}(x ; 1)=1$ and $a_{n}\left(x+e_{1}+e_{2} ; 1\right)=1$

$$
\delta_{n}(1) \leq u_{n}\left(x+e_{1}\right)-u_{n}\left(x+e_{1}+e_{2}\right)-u_{n}\left(x+2 e_{1}\right)+u_{n}\left(x+2 e_{1}+e_{2}\right) \leq 0
$$

where the first inequality is true due to the case assumptions and the optimality of the $v_{n}$ 's and the second one due to the induction hypothesis.

The $v_{n}(x ; 2)$ 's can be proved to satisfy inequality $(2)$ in a similar way. Thus, we can consider $u_{n+1}$ :

$$
\begin{array}{ll} 
& u_{n+1}(x)-u_{n+1}\left(x+e_{2}\right)-u_{n+1}\left(x+e_{1}\right)+u_{n+1}\left(x+e_{1}+e_{2}\right) \\
=\lambda_{1} & {\left[v_{n}(x ; 1)-v_{n}\left(x+e_{2} ; 1\right)-v_{n}\left(x+e_{1} ; 1\right)+v_{n}\left(x+e_{1}+e_{2} ; 1\right)\right]} \\
+\lambda_{2} & {\left[v_{n}(x ; 2)-v_{n}\left(x+e_{2} ; 2\right)-v_{n}\left(x+e_{1} ; 2\right)+v_{n}\left(x+e_{1}+e_{2} ; 2\right)\right]} \\
+x_{1} \mu_{1} & {\left[u_{n}\left(x-e_{1}\right)-u_{n}\left(x+e_{2}-e_{1}\right)-u_{n}(x)+u_{n}\left(x+e_{2}\right)\right]} \\
+\mu_{1} & {\left[u_{n}(x)-u_{n}\left(x+e_{2}\right)-u_{n}(x)+u_{n}\left(x+e_{2}\right)\right]} \\
+x_{2} \mu_{2} & {\left[u_{n}\left(x-e_{2}\right)-u_{n}(x)-u_{n}\left(x+e_{1}-e_{2}\right)+u_{n}\left(x+e_{1}\right)\right]} \\
+\mu_{2} & {\left[u_{n}(x)-u_{n}(x)-u_{n}\left(x+e_{1}\right)+u_{n}\left(x+e_{1}\right)\right]} \\
+\alpha & {\left[u_{n}(x)-u_{n}\left(x+e_{2}\right)-u_{n}\left(x+e_{1}\right)+u_{n}\left(x+e_{1}+e_{2}\right)\right]} \\
\leq & 0
\end{array}
$$

where $\alpha=c \mu_{2}-\left(x_{1}+1\right) \mu_{1}-\left(x_{2}+1\right) \mu_{2}$. The first two terms are less than or equal to 0 since $\delta_{n}(j) \leq 0$, the third, fifth and seventh terms are also non-positive by the induction hypothesis whereas the fourth and sixth terms are 0 . Thus, the value functions, $u_{n}$, satisfy 
inequality (2) for all $n$ whenever $u_{0}$ does.

Intuitively, we expect that it should be more difficult to accept customers when there are many customers already in the system, and so the benefit of the system due to additional customers should decrease with the number of customers in the system. Lemma 1 shows that the benefit due to an additional class $j$ customer, i.e., $D_{n}(j 0)(x)$, is decreasing in the number of class- $i$ customers, $x_{i}, i \neq j$. This is less than our expectation, since we have not shown that the benefit of an additional class- $j$ customer decreases in the number of class- $j$ customers; which corresponds to the concavity of $u_{n}$ in $x_{j}$ for fixed $x_{i}, i \neq j$. We prove the concavity of $u_{n}$ in $x_{2}$ for fixed $x_{1}$ under very restrictive conditions in Section 6 . However, we were not able to prove the concavity of $u_{n}$ for all parameters because of the boundary effects. We also note that concavity of the $u_{n}$ 's would lead to monotonicity of the thresholds. Nevertheless, one easily sees that Lemma 1 guarantees the existence of an optimal threshold policy:

Theorem 1 There exist numbers $\left\{l_{n}^{i}(0), \ldots, l_{n}^{i}(c-1)\right\}_{\{i=1,2\}}$ such that:

$$
\begin{aligned}
& a_{n}(x ; 1)=\left\{\begin{array}{lll}
0 & : & x_{2} \geq l_{n}^{1}\left(x_{1}\right) \\
1 & : & \text { otherwise }
\end{array}\right. \\
& a_{n}(x ; 2)=\left\{\begin{array}{lll}
0 & : & x_{1} \geq l_{n}^{2}\left(x_{2}\right) \\
1 & : & \text { otherwise }
\end{array}\right.
\end{aligned}
$$

\section{Existence of a preferred class}

In this section, we show that under certain conditions customers of one class are admitted to the system whenever there is an idle server; i.e., there exists a preferred class. In determining preferred class(es), the first criterion one thinks of is the well-known $c \mu$ rule, which translates to our system as the $r \mu$ rule. Recall that the $c \mu$ rule is an index rule which always gives priority to a job of class $i^{*}$ with the largest value of $c_{i} \mu_{i}$ among the present jobs, where $c_{i}$ is the unit holding cost and $\mu_{i}$ is average rate of service for class- $i$ customers. $c_{i} \mu_{i}$ is the average cost of having a class- $i$ customer in the system, so the $c \mu$ rule serves the "most expensive" customers first. In our problem, this corresponds to admitting all customers of the class which brings the highest average reward, $r_{i} \mu_{i}$, whenever there is at least one idle server. Under certain conditions, the preferred class is, indeed, determined by the $r \mu$ rule. However, this rule does not hold for all parameters; we present a counterexample in the next section. Another criterion can be the index rule which uses the immediate reward of each class, $R_{i}$; but it is easy to see that if class- 1 customers are slow enough, the optimal policy may reject them even if their immediate rewards are high. As we see later in this section, each of the criteria favors one of the classes, and neither of them determines a preferred class for all possible parameters.

We present the conditions for class 1 to be preferred in terms of average rewards, $r_{i} \mu_{i}$ 's, and for class 2 in terms of immediate rewards, $R_{i}$ 's. We have interpreted the differences $D_{n}(j 0)(x)$ as the net profit of the system due to an additional class- $j$ customer in state $x$. In this section, we concentrate on these differences to show that under certain conditions, they are non-negative; which implies that the corresponding class is preferred. We also 
consider the quantity $D_{n}(12)(x)$, the effect of changing a class- 1 customer in the system to a class- 2 customer in state $x$.

In the proofs of this section, we use, mostly, induction and sample path analysis together. The following $u_{0}$, which is briefly mentioned in Section 2 , satisfies all the statements, allowing us to apply the induction:

$$
u_{0}(x)=x_{1} R_{1}+x_{2} R_{2} \quad \forall x \in \mathcal{S} .
$$

This function corresponds to the assumption that the later rewards of customers, who are still in the system at $n=0$, are collected at $n=0$. We, now, give upper bounds on the differences, $D_{n}(i 0)(x)$ and $D_{n}(12)(x)$ :

Lemma 2 For all $x \in \mathcal{S}$ and for all $n \geq 0$ :

(1) $D_{n}(i 0)(x) \leq R_{i}$ for $i=1,2$.

(2) $D_{n}(12)(x)=-D_{n}(21)(x) \leq R_{1}-R_{2}$.

Proof. We prove the statements by a sample path analysis.

(1) Assume that system $\mathrm{A}$ is in state $x+e_{i}$ and system $\mathrm{B}$ in $x$ when there are $n$ remaining transitions. We let system $\mathrm{A}$ follow the optimal policy, $\pi$, and system $\mathrm{B}$ imitate all the decisions of system $\mathrm{A}$. We couple the two systems via the service and interarrival times, i.e., except for the additional customer in system $\mathrm{A}$, all the departure and arrival times are the same in both systems. We note that system B can always imitate system A since it always has at least as many free servers as system A does. Then, the difference in the expected returns of systems $\mathrm{A}$ and $\mathrm{B}$ is only due to the additional customer in system A:

$$
D_{n}(i 0)(x)=u_{n}\left(x+e_{i}\right)-u_{n}(x) \leq u_{n}\left(x+e_{i}\right)-u_{n}^{\pi}(x)=R_{i} .
$$

where $u_{n}^{\pi}(x)$ is the expected discounted return of system B and $R_{i}$ is the immediate reward of the additional class- $i$ customer in the system, which will be collected eventually due to the definition of $u_{0}$.

(2) Assume that system A starts in state $x+e_{1}$ and system B starts in $x+e_{2}$, where we now couple the additional class- 1 customer, say customer $d_{1}$, in system $\mathrm{A}$ with the additional class-2 customer, say customer $d_{2}$, as well as all other service and interarrival times, so that, as discussed earlier, if $d_{1}$ leaves the system, $d_{2}$ also leaves. Then, we can let system A follow the optimal policy and system B imitate all the decisions of system A. Now, again, the difference in the expected discounted returns of system A and B is only due to the addititonal customers in the beginning:

$$
D_{n}(12)(x)=u_{n}\left(x+e_{1}\right)-u_{n}\left(x+e_{2}\right) \leq u_{n}\left(x+e_{1}\right)-u_{n}^{\pi}\left(x+e_{2}\right)=R_{1}-R_{2},
$$

with $u_{n}^{\pi}\left(x+e_{2}\right)$ the expected discounted return of system B.

Using this lemma, we can present the conditions for class 2 to be preferred:

Theorem 2 If $R_{2} \geq \frac{\lambda_{1}}{\lambda_{1}+\mu_{2}+\beta} R_{1}$, then for all $x \in \mathcal{S}$ and for all $n, D_{n}(20)(x) \geq 0$, hence class 2 is a preferred class. 
Proof. Let $R_{2} \geq \frac{\lambda_{2}}{\lambda_{1}+\mu_{2}+\beta} R_{1}$. We use induction to prove the result. The function $u_{0}$ defined by equation (3) clearly satisfies $D_{0}(20)(x) \geq 0$ for all $x \in \mathcal{S}$. So assume that the statement is also true for period $n$, and consider period $n+1$. Now we use a sample path argument: Let system $\mathrm{A}$ be in state $x+e_{2}$ and system $\mathrm{B}$ in $x$ in period $n+1$. System A rejects all customers in period $n+1$ and system $\mathrm{B}$ takes the optimal actions. Consider an arrival. If system $B$ also rejects either of the two classes, both systems remain in their current states, preserving the extra class-2 customer which leads to a non-negative difference in the rewards of the two systems due to the induction hypothesis. Acceptance of a class-1 customer to system $\mathrm{B}$ leads two systems to two different states $x+e_{2}$ and $x+e_{1}$. If a class- 2 customer is admitted to system $B$, then the two systems couple with no difference in reward. With the departure of the additional class- 2 customer in system $\mathrm{A}$, the systems again enter the same state but with a return of $r_{2}$, whereas all other service completions keep the extra class- 2 customer in system A so that the difference between two systems is at least 0 by the induction hypothesis. Then:

$$
\begin{aligned}
D_{n+1}(20)(x)= & u_{n+1}\left(x+e_{2}\right)-u_{n+1}(x) \\
\geq & \lambda_{1} \min \left\{D_{n}(21)(x), 0\right\}+\lambda_{2} \min \left\{0, D_{n}(20)(x)\right\}+\mu_{2} r_{2} \\
& +(c-1) \mu_{2} \min _{y \in \mathcal{S}}\left\{D_{n}(20)(y)\right\} \\
\geq & \lambda_{1} \min \left\{R_{2}-R_{1}, 0\right\}+\lambda_{2} \times 0+\mu_{2} r_{2} \\
& +\left(1-\lambda_{1}-\lambda_{2}-\mu_{2}-\beta\right) \times 0 \\
\geq & \lambda_{1} \min \left\{R_{2}-R_{1}, 0\right\}+r_{2} \mu_{2}
\end{aligned}
$$

where the first inequality is due to the coupling, the second inequality follows from part 2 of Lemma 2 and uniformization. If $R_{2} \geq R_{1}$, then the statement is proven. Otherwise, we have:

$$
D_{n+1}(20)(x) \geq \lambda_{1}\left(R_{2}-R_{1}\right)+\left(\mu_{2}+\beta\right) R_{2}=\left(\lambda_{1}+\mu_{2}+\beta\right) R_{2}-\lambda_{1} R_{1} \geq 0
$$

where the last inequality is due to the assumption of the theorem.

Thus, whenever $R_{2} \geq \frac{\lambda_{1}}{\lambda_{1}+\mu_{2}+\beta} R_{1}$, class 2 is a preferred class. $R_{2}$ is the immediate reward of class 2. $\frac{\lambda_{1}}{\lambda_{1}+\mu_{2}+\beta}$ is the probability that a class-1 customer arrives before the system closes down or a class- 2 customer finishes service. Then, if the immediate reward of a class- 2 customer is higher than the expected reward of waiting for the next class-1 customer, it is more profitable to allocate one of the available servers to an arriving class2 customer instead of reserving it for a future class- 1 customer. We have the following corollary of this result:

Corollary 1 Whenever $r_{2} \geq r_{1}$ so that class-2 customers bring higher rewards, and require shorter service times, class-2 customers are preferred.

We derive a similar condition for class 1 to be preferred. However, this requires some more work, since we have to consider a lower bound on the effect of changing a class-1 customer to a class- 2 customer as well as the effect of an additional class- 1 customer. 
Lemma 3 If $r_{1} \mu_{1} \geq \frac{\lambda_{2}}{\lambda_{2}+\mu_{2}+\beta} r_{2} \mu_{2}$, then for all $x \in \mathcal{S}$ and for all $n$ :

(1) $D_{n}(10)(x) \geq 0$.

(2) $D_{n}(12)(x) \geq \frac{r_{1} \mu_{1}-r_{2} \mu_{2}}{\mu_{2}+\beta}$.

Proof. We use induction on the number of transitions, $n$. Both statements are satisfied for $u_{0}$ defined by (3). Assume that both are true for $n$. Now we have to consider two pairs of systems, one for $D_{n+1}(10)(x)$ and the other for $D_{n+1}(12)(x)$.

(1) Consider the first pair: Assume that system $\mathrm{A}$ is in state $x+e_{1}$ and system $\mathrm{B}$ is in $x$ in period $n+1$, and we couple the two systems in such a way that system $\mathrm{A}$ rejects all customers in period $n+1$, whereas system $\mathrm{B}$ follows the optimal policy. If upon an arrival system $B$ also rejects either of the two classes, both systems remain in their current states, preserving the extra class- 1 customer which leads to a non-negative difference in the rewards of the systems due to the induction hypothesis. Acceptance of a class-1 customer to system $B$ leads both systems to enter the same state with no difference in reward. If a class-2 customer is admitted to system $\mathrm{B}$, then the systems move to two different states $x+e_{1}$ and $x+e_{2}$. With the departure of the additional class- 1 customer in system $\mathrm{A}$, the systems again enter the same state but with a return of $r_{1}$, whereas all other service completions keep the difference between the two systems the same, i.e., the only difference is the extra class- 1 customer, so that the difference between the two systems is at least 0 by the induction hypothesis. Then:

$$
\begin{aligned}
D_{n+1}(10)(x) \geq & \lambda_{1} \min \left\{D_{n}(10)(x), 0\right\}+\lambda_{2} \min \left\{D_{n}(10)(x), D_{n}(12)(x)\right\}+\mu_{1} r_{1} \\
& +\left(c \mu_{2}-\mu_{1}\right) \min _{y \in \mathcal{S}}\left\{D_{n}(10)(y)\right\} \\
\geq & \lambda_{1} \times 0+\lambda_{2} \min \left\{0, D_{n}(12)(x)\right\}+\mu_{1} r_{1} \\
& +\left(1-\lambda_{1}-\lambda_{2}-\mu_{1}-\beta\right) \times 0 \\
\geq & \lambda_{2} \min \left\{\frac{r_{1} \mu_{1}-r_{2} \mu_{2}}{\mu_{2}+\beta}, 0\right\}+r_{1} \mu_{1}
\end{aligned}
$$

where the first inequality is due to coupling, the second due to the induction hypothesis for $D_{n}(10)(x)$ and uniformization, and finally the third one follows from the induction hypothesis for $D_{n}(12)(x)$. If $r_{1} \mu_{1} \geq r_{2} \mu_{2}$, the statement is proven; otherwise:

$$
D_{n+1}(10)(x) \geq r_{1} \mu_{1} \frac{\lambda_{2}+\mu_{2}+\beta}{\mu_{2}+\beta}-\frac{\lambda_{2} r_{2} \mu_{2}}{\mu_{2}+\beta} \geq 0
$$

where the last inequality is due to the assumption of the theorem. Thus, the first statement is true for all $x \in \mathcal{S}$ and for all $n \geq 0$.

(2) Now consider the second pair of systems: Let system $\mathrm{A}^{\prime}$ be in state $x+e_{1}$ and system $\mathrm{B}^{\prime}$ in $x+e_{2}$ in period $n+1$. System $\mathrm{B}^{\prime}$ takes the optimal actions and system $\mathrm{A}^{\prime}$ imitates all the actions of system $B^{\prime}$ in this period. We, as in Lemma 2, couple the additional class-2 customer, say customer $d_{2}$, in system $\mathrm{B}^{\prime}$ with the additional class-1 customer, say customer $d_{1}$ in system $A^{\prime}$, as well as all other service and interarrival times. Then, if $d_{1}$ leaves the system, which happens with probability $\mu_{1}, d_{2}$ also leaves. The departure of $d_{1}$ leads the system to couple with a reward of $r_{1}-r_{2}$, the departure of $d_{2}$ alone, which happens with probability $\mu_{2}-\mu_{1}$, takes the systems to two different states, $x+e_{1}$ and $x$ with a reward 
of $-r_{2}$ and whenever there is any other transition, both systems continue to have their additional customers so that the difference between the two systems is due to changing a class-1 customer to class 2 :

$$
\begin{aligned}
D_{n+1}(12)(x) \geq & \mu_{1}\left(r_{1}-r_{2}\right)+\left(\mu_{2}-\mu_{1}\right)\left(-r_{2}+D_{n}(10)(x)\right) \\
& +\left(\lambda_{1}+\lambda_{2}+(c-1) \mu_{2}\right) \min _{y \in \mathcal{S}}\left\{D_{n}(12)(y)\right\} \\
\geq & r_{1} \mu_{1}-r_{2} \mu_{2}+\left(1-\mu_{2}-\beta\right) \frac{r_{1} \mu_{1}-r_{2} \mu_{2}}{\mu_{2}+\beta} \\
= & \frac{r_{1} \mu_{1}-r_{2} \mu_{2}}{\mu_{2}+\beta}
\end{aligned}
$$

where the first inequality is due to the coupling and the second follows by uniformization and the induction hypotheses for both $D_{n}(10)(x)$ and $D_{n}(12)(x)$. This proves the second part of the lemma.

This lemma immediately leads to the following theorem which gives the sufficient conditions for class 1 to be preferred:

Theorem 3 If $r_{1} \mu_{1} \geq \frac{\lambda_{2}}{\lambda_{2}+\mu_{2}+\beta} r_{2} \mu_{2}$, then class 1 is a preferred class.

\section{A discussion on preferred class and a counterexample}

\subsection{Non-existence of a preferred class}

In the previous section, we give sufficient conditions for each class to be preferred. It is easily observed that these conditions are not complementary, i.e., for certain values of parameters our results cannot claim that there exists a preferred class:

Remark 1 If the parameter values are such that:

$$
\frac{\lambda_{2}+\mu_{2}+\beta}{\lambda_{2}}<\frac{r_{2} \mu_{2}}{r_{1} \mu_{1}}<\frac{\lambda_{1}\left(\mu_{2}+\beta\right)}{\left(\lambda_{1}+\mu_{2}+\beta\right)\left(\mu_{1}+\beta\right)},
$$

then our results are inconclusive.

We consider a control problem, so it is more likely to consider the system at an operational level, rather than at a strategical or tactical level. This requires $\beta$ to be small $(\beta<0.001)$, so that for many parameter values the first term in $(4)$ will be larger than the third implying that a preferred class does exist. Whenever there is not a preferred class, we know little about the optimal policy: We still have a threshold policy, and it is also easy to show that the optimal policy has to use all the servers. However, our results, so far, do not guarantee that in each state at least one of the two classes has to be accepted. Of course, this is very counterintuitive, and we conjecture that it is never optimal to reject both of the classes in any state:

Conjecture 1 For all parameter values, there exists no state in which both classes are rejected. 
It is easier to believe that the system preference may change dynamically, so that it may be optimal to accept either of the classes in some states and reject in the others, i.e., there is not a preferred class. However, we do not believe this:

Conjecture 2 There always exists a preferred class.

\subsection{A counterexample to the $r \mu$ rule}

We present a counterexample to the $r \mu$ rule in which class- 2 customers are rejected in two of the states, whereas class 1 is accepted in all states, even if the average reward of class 2 is slightly higher than that of class 1 . The system has 6 servers. The parameter values are as follows, before normalization: $\lambda_{1}=3, \lambda_{2}=0.01, \mu_{1}=0.5, \mu_{2}=5, r_{1}=2, r_{2}=0.202$ and $\beta=0$ so that $r_{1} \mu_{1}=1$ and $r_{2} \mu_{2}=1.01$. The optimal policy accepts class- 1 customers in all states, so class 1 is the preferred class in this example. However, class-2 customers are rejected in states $(1,4)$ and $(0,5)$.

\subsection{Sufficient conditions for the $r \mu$ rule}

From the results of the previous section, we can easily derive sufficient conditions for the $r \mu$ rule to determine the preferred class:

\section{Corollary 2}

$$
\begin{aligned}
& \text { If } \frac{\lambda_{1}\left(\mu_{2}+\beta\right)}{\left(\lambda_{1}+\mu_{2}+\beta\right)\left(\mu_{1}+\beta\right)} \leq 1, \text { then the preferred class is determined by the } \\
& \text { rurule. }
\end{aligned}
$$

Proof. If $r_{2} \mu_{2} \geq r_{1} \mu_{1}$ :

$$
\frac{r_{2} \mu_{2}}{r_{1} \mu_{1}} \geq 1 \geq \frac{\lambda_{1}\left(\mu_{2}+\beta\right)}{\left(\lambda_{1}+\mu_{2}+\beta\right)\left(\mu_{1}+\beta\right)}
$$

so that class 2 is preferred by Theorem 2 .

If $r_{1} \mu_{1} \geq r_{2} \mu_{2}$, then:

$$
\frac{r_{2} \mu_{2}}{r_{1} \mu_{1}} \leq 1<\frac{\lambda_{2}+\mu_{2}+\beta}{\lambda_{2}}
$$

which implies that class- 1 customers are preferred by Theorem 3 .

Note that this corollary gives the sufficient conditions. So, even if the given condition is not satisfied, the class with higher $r_{i} \mu_{i}$ might still be preferred.

\section{Concavity of the value functions under certain conditions}

The system cannot have more than $c$ customers at any time. Thus, as the number of class$i$ customers increases in the system, we expect that thresholds on the number of class $-j$, $i \neq j$, customers decreases. This is, in fact, what we have observed in all the examples we have worked out and it corresponds to the concavity of the $u_{n}$ 's in $x_{i}$ for fixed $x_{j}, j \neq i$. 
However, it is difficult to prove the concavity in full generality because of the boundary effects and state-dependent service rates. We derive explicit expressions for bounds on the differences due to an additional customer, $D_{n}(j 0)(x)$, and due to changing the class of a customer, $D_{n}(i j)(x)$, to show the concavity of the $u_{n}$ 's in $x_{2}$ only under some restrictive conditions on the parameters:

$$
\begin{array}{ll}
\text { Condition 1: } & \frac{r_{2} \mu_{2}}{r_{1} \mu_{1}} \leq \frac{\lambda_{1}}{\lambda_{1}+\mu_{1}+\beta} \& \\
& \lambda_{1}\left(\mu_{2}-\mu_{1}\right) \leq\left(\mu_{1}+\beta\right)\left(\mu_{2}+\beta\right)\left(1+\frac{\mu_{2}+\beta}{\lambda_{1}}\right) \\
\text { Condition 2: } & \frac{\lambda_{1}}{\lambda_{1}+\mu_{1}+\beta} \leq \frac{r_{2} \mu_{2}}{r_{1} \mu_{1}} \leq \frac{\lambda_{1}}{\left(\lambda_{1}+\mu_{2}+\beta\right)} \frac{\mu_{2}+\beta}{\left(\mu_{1}+\beta\right)} \& \\
& \lambda_{1}\left(\mu_{2}-\mu_{1}\right) \leq\left(\mu_{1}+\beta\right)\left(\mu_{2}+\beta\right)\left(1+\frac{\mu_{2}+\beta}{\lambda_{1}+\lambda_{2}}\right)
\end{array}
$$

We first observe that under either of these conditions class 1 is a preferred class. This is obvious for Condition 1 (see Theorem 3). Now consider Condition 2. It can be easily shown that the second part of Condition 2 is equivalent to:

$$
\frac{\lambda_{1}}{\left(\lambda_{1}+\mu_{2}+\beta\right)} \frac{\mu_{2}+\beta}{\left(\mu_{1}+\beta\right)} \leq 1+\frac{\left(\mu_{2}+\beta\right)^{2}}{\left(\lambda_{1}+\lambda_{2}\right)\left(\lambda_{1}+\mu_{2}+\beta\right)}
$$

But then we have:

$$
\frac{\lambda_{1}\left(\mu_{2}+\beta\right)}{\left(\mu_{1}+\beta\right)\left(\lambda_{1}+\mu_{2}+\beta\right)} \leq 1+\frac{\left(\mu_{2}+\beta\right)^{2}}{\left(\lambda_{1}+\lambda_{2}\right)\left(\lambda_{1}+\mu_{2}+\beta\right)} \leq 1+\frac{\mu_{2}+\beta}{\lambda_{1}+\lambda_{2}} \leq 1+\frac{\mu_{2}+\beta}{\lambda_{2}} .
$$

Thus when Condition 2 is satisfied, we have:

$$
r_{2} \mu_{2} \leq \frac{\lambda_{2}+\mu_{2}+\beta}{\lambda_{2}} r_{1} \mu_{1}
$$

which implies that class 1 is preferred by Theorem 3. Moreover, it is easily observed from Theorem 2 that class 2 is also preferred under Condition 2. Now we can present the following result:

Lemma 4 Assume that either Condition 1 or Condition 2 is satisfied. Then, for all $x \in \mathcal{S}$ and for all $n \geq 0$ :

(1) $u_{n}(x)$ is concave in $x_{2}$ for each fixed $x_{1}$, i.e., $D_{n}(20)\left(x+e_{2}\right) \leq D_{n}(20)(x)$.

(2) $R_{2}-\frac{\lambda_{1}}{\lambda_{1}+\mu_{2}+\beta} R_{1} \leq D_{n}(20)(x)$.

The proof of this lemma is very technical, which requires to derive explicit expressions of a lower bound on $D_{n}(20)(x)$ and an upper bound on $D_{n}(21)(x)$. Conclusions of the previous sections do not need these explicit forms, which are very complicated in sight and bring no intuition at all. Thus, we exclude them from the main body of the paper. We present the proof of this lemma along with another lemma in the Appendix. Proofs of both lemmas are based on induction, and they are conceptually straightforward although very tedious. 
Under Condition 2, both class 1 and 2 are preferred so that thresholds for both classes are trivial, i.e., $l_{n}^{j}(k)=c-k$ for all $k=0, . ., c-1$ for $j=1,2$. For Condition 1 , concavity of the $u_{n}$ 's in $x_{2}$ guarantees the monotonicity of the thresholds:

Proposition 1 Assume that Condition 1 holds. Then, $l_{n}^{2}(k)$ is decreasing in $k$.

Proof. By definition of $l_{n}^{2}(k)$, we have $a_{n}\left(l_{n}^{2}(k), k ; 2\right)=0$. Then:

$$
u_{n}\left(l_{n}^{2}(k), k+2\right)-u_{n}\left(l_{n}^{2}(k), k+1\right) \leq u_{n}\left(l_{n}^{2}(k), k+1\right)-u_{n}\left(l_{n}^{2}(k), k\right)<0
$$

where the first inequality is due to $a_{n}\left(l_{n}^{2}(k), k ; 2\right)=0$ and the second is due to Lemma 4 . But then by definition of $l_{n}^{2}(k+1), l_{n}^{2}(k+1) \leq l_{n}^{2}(k)$.

\section{Generalizations and Future Research}

Our results still hold when the arrival process is a Markov arrival process (MAP) instead of a Poisson process, see Örmeci (1998) for details. MAPs are defined by Asmussen \& Koole (1993) who have also shown that any independent arrival process with multiple classes of customers can be approximated arbitrarily closely by an MAP. The MAPs bring two main benefits: One is to be able to model the departure process of most queueing systems with exponentially distributed sojourn time, which can then be used as input to the loss system we consider. For example, we can define an appropriate MAP to reproduce the main result of $\mathrm{Ku} \&$ Jordan (1997). Secondly, the MAPs can model many generalizations of the exponential distribution, e.g., phase-type renewal process and Markov Modulated Poisson Process (see Hordijk \& Koole (1993)).

We can also consider the system under a general arrival process, which can be modeled as an embedded MDP at arrival epochs. This will generalize the MAPs.

A system with more than two classes is another natural extension to be considered. The arguments used here to determine preferred class(es) can still be used when the number of classes is increased, but the sufficient conditions for a class to be preferred will consist of relations between the rewards and service rates of this class and all the other classes. Moreover, existence of thresholds, in this case, is very difficult to establish. As a result, the extension of our results to systems with more classes is not obvious, and so worthwhile to explore.

We have also considered the system under batch arrivals (see Örmeci \& Burnetas (1999)). Although some of the results can be replicated exactly for batch arrivals, they are not enough to characterize the optimal policy in terms of thresholds. However, we can still show the existence of a preferred class under certain conditions.

In this paper, we assume that customers of each class bring fixed rewards. In Örmeci, Burnetas \& Emmons (1999), we have considered random rewards for each class. We have shown the existence of thresholds. Under random rewards, we cannot specify one class as preferred, since the reward of each customer, even if they are from the same class, varies. However, it is shown that there exist preferred customers under certain conditions, where preferred customers of each class are specified as the customers who bring at least a certain amount of reward. 
Assuming fixed rewards for each class also implies that we have no control over the rewards. However, pricing has been an important issue for the last decades. Thus, we can reverse the roles in the system we have considered so that we do not have the control on the admission but on the reward of an incoming customer, which gives us an indirect admission control. Miller \& Buckman (1987) have considered a static transfer pricing problem for one class in an $M / M / c / c$ system which serves as a model of a service department. More general pricing schemes can be considered, e.g. dynamic pricing can be also allowed as opposed to static pricing.

\section{References}

Asmussen, S. \& Koole, G. M. (1993), 'Marked point processes as limits of markovian arrivals streams', Jour. of Appl. Probab. 30, 365-372.

Baras, J., Dorsey, A. \& Makowski, A. (1985), ' $k$ competing queues with geometric service requirements and linear costs: The $\mu c$ rule is always optimal', Syst. Control Lett. 6, 173-180.

Buyukkoc, C., Varaiya, P. \& Walrand, J. (1985), 'The $c \mu$ rule revisited', Adv. in Appl. Prob. 17, 237-238.

Carrizosa, E., Conde, E. \& Munoz-Marquez, M. (1998), 'Admission policies in loss queueing models with heterogeneous arrivals', Management Science 44, 311-320.

Hordijk, A. \& Koole, G. (1993), 'On the optimality of lept and $\mu c$ rules for parallel processors and dependent arrival processes', Adv. Appl. Probab. 25, 979-996.

$\mathrm{Ku}, \mathrm{C}$. \& Jordan, S. (1997), 'Access control to two multiserver loss queues in series', IEEE Transactions on Automatic Control 42, 1017-1023.

Lippman, S. A. \& Ross, S. M. (1971), 'The streetwalker's dilemma: A job shop model', SIAM J. Appl. Math. 20, 336-342.

Miller, B. (1971), 'A queuing reward system with several customer classes', Management Science 16, 234-245.

Miller, B. L. \& Buckman, A. G. (1987), 'Cost allocation and opportunity costs', Management Science 33, 626-639.

Örmeci, E. L. (1998), Idling Rules for Queues with Preferred Customers, Ph.D. thesis, Case Western Reserve University, Cleveland.

Örmeci, E. L. \& Burnetas, A. (1999), Admission Policies for a Two Class Loss System with Batch Arrivals, EURANDOM Technical Report 99-052, Eindhoven.

Örmeci, E. L., Burnetas, A. \& Emmons, H. (1999), Admission Policies for a Two Class Loss System with Random Rewards, EURANDOM Technical Report 99-051, Eindhoven.

Ross, K. W. (1995), Multiservice Loss Models for Broadband Telecommunication Networks, Springer-Verlag, Great Britain. 
Varaiya, P. P., Walrand, J. C. \& Buyukkoc, C. (1985), 'Extensions of the multi-armed bandit problem', IEEE Transactions on Automatic Control 30, 426-439.

Walrand, J. (1988), Introduction to Queueing Networks, Prentice Hall, Englewood Cliffs, N.J.

\section{Appendix}

We want to prove Lemma 4. For this, we first define $D_{n}^{\min }(i j)$ and $D_{n}^{\max }(i j)$ as a lower and an upper bound, respectively, on the differences $D_{n}(i j)(x)$, so that for all $n$ :

$$
D_{n}^{\min }(i j) \leq D_{n}(i j)(x) \leq D_{n}^{\max }(i j) \quad \forall x \in \mathcal{S}
$$

Now, we need to prove the following lemma, which will be used in the proof of Lemma 4.

\section{Lemma 5}

(1) If $\frac{\lambda_{1}}{\lambda_{1}+\mu_{1}+\beta} \leq \frac{r_{2} \mu_{2}}{r_{1} \mu_{1}} \leq \frac{\lambda_{2}+\mu_{2}+\beta}{\lambda_{2}}$, then for all $x \in \mathcal{S}$, for all $n \geq 0$ :

$$
\begin{aligned}
& D_{n}^{\text {min }}(12)=\frac{r_{1} \mu_{1}\left(\lambda_{1}+\lambda_{2}+\mu_{2}+\beta\right)-r_{2} \mu_{2}\left(\lambda_{1}+\lambda_{2}+\mu_{1}+\beta\right)}{\left(\lambda_{1}+\lambda_{2}+\mu_{2}+\beta\right)\left(\mu_{1}+\beta\right)+\lambda_{1}\left(\mu_{2}-\mu_{1}\right)} \leq D_{n}(12)(x) \\
& \& 0 \leq D_{n}^{\text {min }}(10)=\frac{r_{1} \mu_{1}\left(\lambda_{2}+\mu_{2}+\beta\right)-r_{2} \mu_{2} \lambda_{2}}{\left(\lambda_{1}+\lambda_{2}+\mu_{2}+\beta\right)\left(\mu_{1}+\beta\right)+\lambda_{1}\left(\mu_{2}-\mu_{1}\right)} \leq D_{n}(10)(x) . \\
& \text { (2) If } \frac{r_{2} \mu_{2}}{r_{1} \mu_{1}} \leq \frac{\lambda_{1}}{\lambda_{1}+\mu_{1}+\beta} \text {, then for all } x \in \mathcal{S} \text { for all } n \geq 0: \\
& D_{n}^{\text {min }}(12)=\frac{r_{1} \mu_{1}\left(\lambda_{1}+\mu_{2}+\beta\right)}{\left(\lambda_{1}+\mu_{1}+\beta\right)\left(\mu_{2}+\beta\right)}-\frac{r_{2} \mu_{2}}{\mu_{2}+\beta} \leq D_{n}(12)(x) \text { and } \\
& 0 \leq D_{n}^{\text {min }}(10)=\frac{r_{1} \mu_{1}}{\lambda_{1}+\mu_{1}+\beta} \leq D_{n}(10)(x) \text {. }
\end{aligned}
$$

Proof. We only give a sketch of the proof: We consider the differences $D_{n}(12)(x)$ and $D_{n}(10)(x)$ simultaneously, and use the same sample path analysis as in the proof of Lemma 3. We consider two pairs of systems at the same time, one pair for $D_{n}(12)(x)$ and the other for $D_{n}(10)(x) . D_{n}^{\min }(12)$ and $D_{n}^{\min }(10)$ are lower bounds for $D_{n}(12)(x)$ and $D_{n}(10)(x)$, respectively, whereas in Lemma 3 , lower bound for $D_{n}(10)(x)$ is 0 , and for $D_{n}(12)(x)$ it is $\frac{r_{1} \mu_{1}-r_{2} \mu_{2}}{\mu_{2}+\beta}$. Thus, in this lemma, both lower bounds have more complicated expressions under each of the conditions. However, this does not affect the method of the proof, so that after replacing these lower bounds appropriately, the proof proceeds exactly in the same way as Lemma 3.

Now, we are ready to prove Lemma 4:

Lemma 4 Assume that either Condition 1 or Condition 2 is satisfied. Then, for all $x \in \mathcal{S}$ and for all $n \geq 0$ :

$$
\begin{aligned}
& \text { (1) } u_{n}(x) \text { is concave in } x_{2} \text { for each fixed } x_{1} \text {, i.e., } \\
& D_{n}(20)\left(x+e_{2}\right) \leq D_{n}(20)(x) \text {. } \\
& \text { (2) } R_{2}-\frac{\lambda_{1}}{\lambda_{1}+\mu_{2}+\beta} R_{1} \leq D_{n}(20)(x)
\end{aligned}
$$


Proof. We show both statements together by induction on the remaining number of transitions under either of the two conditions. Let $u_{0}$ be defined by (3). Then, all claims are true for $n=0$. Now assume for $n$.

Assume that Condition 1 is true. We first show that $v_{n}(. ; j)$ 's also satisfy the monotonicity in part (1). Consider $v_{n}(. ; 1)$ 's. We differentiate the cases when all the servers are occupied and when there is at least one free server. If $x_{1}+x_{2}+2<c$ :

$$
\begin{aligned}
v_{n}\left(x+2 e_{2} ; 1\right)-v_{n}\left(x+e_{2} ; 1\right) & =u_{n}\left(x+e_{1}+2 e_{2}\right)-u_{n}\left(x+e_{1}+e_{2}\right) \\
& \leq u_{n}\left(x+e_{1}+e_{2}\right)-u_{n}\left(x+e_{1}\right) \\
& =v_{n}\left(x+e_{2} ; 1\right)-v_{n}(x ; 1)
\end{aligned}
$$

where the inequality follows from the induction hypothesis and the rest is due to the fact that class 1 is preferred under either of the assumed conditions. If $x_{1}+x_{2}+2=c$, we consider the right and left hand sides of the inequality separately:

$$
\begin{aligned}
v_{n}\left(x+e_{2} ; 1\right)-v_{n}(x ; 1) & =u_{n}\left(x+e_{1}+e_{2}\right)-u_{n}\left(x+e_{1}\right) \geq D_{n}^{\min }(20) \\
v_{n}\left(x+2 e_{2} ; 1\right)-v_{n}\left(x+e_{2} ; 1\right) & =u_{n}\left(x+2 e_{2}\right)-u_{n}\left(x+e_{1}+e_{2}\right) \\
& \leq D_{n}^{\max }(21)
\end{aligned}
$$

by definition of $D_{n}^{\max }(i j)$ and $D_{n}^{\min }(i j)$. Thus if $D_{n}^{\min }(20) \geq D_{n}^{\max }(21)$, then the desired inequality is satisfied. From Lemma 5, part (2) (note that $D_{n}^{\max }(21)=-D_{n}^{\min }(12)$ ), $D_{n}^{\min }(20) \geq D_{n}^{\max }(21)$ if and only if

$$
\begin{aligned}
\frac{\lambda_{1} r_{1} \mu_{1}}{\left(\mu_{1}+\beta\right)\left(\lambda_{1}+\mu_{2}+\beta\right)} \leq \frac{r_{1} \mu_{1}\left(\lambda_{1}+\mu_{2}+\beta\right)}{\left(\lambda_{1}+\mu_{1}+\beta\right)\left(\mu_{2}+\beta\right)} & \Longleftrightarrow \\
\lambda_{1}\left(\lambda_{1}+\mu_{1}+\beta\right)\left(\mu_{2}+\beta\right) \leq\left(\lambda_{1}+\mu_{2}+\beta\right)^{2}\left(\mu_{1}+\beta\right) & \Longleftrightarrow \\
0 \leq\left(\mu_{2}+\beta\right)\left(\mu_{1}+\beta\right)\left(\lambda_{1}+\mu_{2}+\beta\right)-\lambda_{1}^{2}\left(\mu_{2}-\mu_{1}\right) &
\end{aligned}
$$

which is true by the second part of Condition 1.

Now we consider $v_{n}(. ; 2)$ 's. Due to the induction hypothesis, we can have only three different cases for $a_{n}\left(x+2 e_{2} ; 2\right)$ and $a_{n}(x ; 2)$ since $a_{n}(x ; 2)=0$ and $a_{n}\left(x+2 e_{2} ; 2\right)=1$ is not allowed. When the actions are the same for both states $\left(x+2 e_{2}\right)$ and $x$, the statement is obviously true by the induction hypothesis. Hence we need to consider only the case with $a_{n}(x ; 2)=1$ and $a_{n}\left(x+2 e_{2} ; 2\right)=0$ :

$$
\begin{aligned}
v_{n}(x ; 2)-v_{n}\left(x+e_{2} ; 2\right)-v_{n}\left(x+e_{2} ; 2\right)+v_{n}\left(x+2 e_{2} ; 2\right) & \leq \\
u_{n}\left(x+e_{2}\right)-u_{n}\left(x+e_{2}\right)-u_{n}\left(x+2 e_{2}\right)+u_{n}\left(x+2 e_{2}\right) & =0
\end{aligned}
$$

where the inequality follows from the optimality of $v_{n}$ 's.

Now we can consider $u_{n+1}$ 's:

$$
\begin{aligned}
& u_{n+1}(x)-2 u_{n+1}\left(x+e_{2}\right)+u_{n+1}\left(x+2 e_{2}\right) \\
= & \lambda_{1}\left[v_{n}(x ; 1)-2 v_{n}\left(x+e_{2} ; 1\right)+v_{n}\left(x+2 e_{2} ; 1\right)\right] \\
+ & \lambda_{2}\left[v_{n}(x ; 2)-2 v_{n}\left(x+e_{2} ; 2\right)+v_{n}\left(x+2 e_{2} ; 2\right)\right] \\
+ & x_{1} \mu_{1}\left[u_{n}\left(x-e_{1}\right)-2 u_{n}\left(x-e_{1}+e_{2}\right)+u_{n}\left(x-e_{1}+2 e_{2}\right)\right] \\
+ & x_{2} \mu_{2}\left[u_{n}\left(x-e_{2}\right)-2 u_{n}(x)+u_{n}\left(x+e_{2}\right)\right]
\end{aligned}
$$




$$
\begin{aligned}
& +\mu_{2}\left[u_{n}(x)-2 u_{n}(x)+u_{n}\left(x+e_{2}\right)\right] \\
& +\mu_{2}\left[u_{n}(x)-2 u_{n}\left(x+e_{2}\right)+u_{n}\left(x+e_{2}\right)\right] \\
& +\left[\left(c-x_{1}-x_{2}-2\right) \mu_{2}+x_{1}\left(\mu_{2}-\mu_{1}\right)\right]\left[u_{n}(x)-2 u_{n}\left(x+e_{2}\right)+u_{n}\left(x+2 e_{2}\right)\right] \geq 0
\end{aligned}
$$

where the first two terms are non-positive since we have shown that $v_{n}$ 's satisfy the given inequality, the third, fourth and seventh terms are non-positive by the induction hypothesis and fifth and sixth terms cancel out each other. Thus part (1) is true for all $n$ under Condition 1.

Now we show that part (2) is also true under Condition 1 . For $v_{n}(. ; 1)$, we have:

$$
\begin{aligned}
& v_{n}\left(x+e_{2} ; 1\right)-v_{n}(x ; 1) \geq \min \left\{u_{n}\left(x+e_{1}+e_{2}\right)-u_{n}\left(x+e_{1}\right),\right. \\
&\left.u_{n}\left(x+e_{2}\right)-u_{n}\left(x+e_{1}\right)\right\} \\
& \geq \min \left\{D_{n}^{\min }(20), D_{n}^{\min }(21)\right\}=D_{n}^{\min }(21),
\end{aligned}
$$

where the first inequality is due the fact that $a_{n}(x ; 1)=1$ whenever $x_{1}+x_{2}<c$ and $a_{n}(x ; 1)=0$ if $x_{1}+x_{2}=c$, the second is due to definitions of $D_{n}^{\min }(20)$ and $D_{n}^{\min }(12)$ and finally the last equality follows since $D_{n}^{\min }(21)=-D_{n}^{\max }(12)=R_{2}-R_{1} \leq D_{n}^{\min }(20)$ by Lemma 2 , which is easily verified.

For $j=2$, we first observe that $D_{n}^{\min }(20) \leq 0$ :

$$
\frac{r_{2} \mu_{2}}{\mu_{2}+\beta}-\frac{\lambda_{1} r_{1} \mu_{1}}{\left(\lambda_{1}+\mu_{2}+\beta\right)\left(\mu_{1}+\beta\right)} \leq 0
$$

by the assumption on $r_{2} \mu_{2} / r_{1} \mu_{1}$. Then:

$$
\begin{aligned}
& v_{n}\left(x+e_{2} ; 2\right)-v_{n}(x ; 2) \geq \min \left\{u_{n}\left(x+e_{2}\right)-u_{n}(x), u_{n}\left(x+e_{2}\right)-u_{n}\left(x+e_{2}\right),\right. \\
&\left.u_{n}\left(x+2 e_{2}\right)-u_{n}\left(x+e_{2}\right)\right\} \\
& \geq \min \left\{D_{n}^{\min }(20), 0\right\}=D_{n}^{\min }(20)
\end{aligned}
$$

where the first inequality is due to statement (1) which does not allow $a_{n}(x ; 2)=0$ and $a_{n}\left(x+e_{2} ; 2\right)=1$ simultaneously, the second inequality is by definition of $D_{n}^{\min }(20)$ and last equality follows from the above observation, $D_{n}^{\min }(20) \leq 0$. Then, for $u_{n+1}$, we have:

$$
\begin{aligned}
D_{n+1}(20)(x) \geq & r_{2} \mu_{2}+\lambda_{1} D_{n}^{\min }(21)+\lambda_{2} D_{n}^{\min }(20)+(c-1) \mu_{2} D_{n}^{\min }(20) \\
= & r_{2} \mu_{2}+\lambda_{1}\left[\frac{r_{2} \mu_{2}}{\mu_{2}+\beta}-\frac{r_{1} \mu_{1}}{\mu_{1}+\beta}\right]+\left(1-\lambda_{1}-\mu_{2}-\beta\right) D_{n}^{\min }(20) \\
= & D_{n}^{\text {min }}(20)+\frac{\left(\lambda_{1}+\mu_{2}+\beta\right) r_{2} \mu_{2}}{\mu_{2}+\beta}-\frac{\lambda_{1} r_{1} \mu_{1}}{\mu_{1}+\beta} \\
& -\left(\lambda_{1}+\mu_{2}+\beta\right) D_{n}^{\min }(20) \\
= & D_{n}^{\text {min }}(20)
\end{aligned}
$$

where all relations follow from the induction hypothesis, uniformization and some algebra.

Thus, both statements are proven under Condition 1.

Now suppose that Condition 2 is satisfied. We only need to show that we still have $D_{n}^{\min }(20) \geq D_{n}^{\max }(21)$ since the rest of the proof depends only on the observation that class 
1 is a preferred class under either of the conditions. Under Condition 2, Lemma 5, part (2) implies that $D_{n}^{\min }(20) \geq D_{n}^{\max }(21)$ if and only if

$$
\begin{aligned}
R_{1} \frac{\lambda_{1}}{\lambda_{1}+\mu_{2}+\beta}-R_{2} \leq \frac{r_{1} \mu_{1}\left(\lambda_{1}+\lambda_{2}+\mu_{2}+\beta\right)-r_{2} \mu_{2}\left(\lambda_{1}+\lambda_{2}+\mu_{1}+\beta\right)}{\left(\lambda_{1}+\lambda_{2}+\mu_{2}+\beta\right)\left(\mu_{1}+\beta\right)+\lambda_{1}\left(\mu_{2}-\mu_{1}\right)} & \Leftrightarrow \\
R_{2} \lambda_{2}\left(\mu_{2}-\mu_{1}\right) \leq & R_{1}\left[\frac{\left(\lambda_{1}+\lambda_{2}+\mu_{2}+\beta\right)\left(\mu_{1}+\beta\right)\left(\mu_{2}+\beta\right)-\lambda_{1}^{2}\left(\mu_{2}-\mu_{1}\right)}{\lambda_{1}+\mu_{2}+\beta}\right] \\
\frac{R_{2}}{R_{1}} \leq \frac{\left(\lambda_{1}+\lambda_{2}+\mu_{2}+\beta\right)\left(\mu_{1}+\beta\right)\left(\mu_{2}+\beta\right)-\lambda_{1}^{2}\left(\mu_{2}-\mu_{1}\right)}{\lambda_{2}\left(\lambda_{1}+\mu_{2}+\beta\right)\left(\mu_{2}-\mu_{1}\right)} & \Leftrightarrow
\end{aligned}
$$

If the right hand side of the inequality is greater than the quantity $\lambda_{1}\left(\mu_{2}+\beta\right) /\left(\lambda_{1}+\mu_{2}+\right.$ $\beta)\left(\mu_{1}+\beta\right)$, then the above inequality is always satisfied by the first part of Condition 2 :

$$
\begin{aligned}
\frac{\lambda_{1}}{\lambda_{1}+\mu_{2}+\beta} \leq \frac{\left(\lambda_{1}+\lambda_{2}+\mu_{2}+\beta\right)\left(\mu_{1}+\beta\right)\left(\mu_{2}+\beta\right)-\lambda_{1}^{2}\left(\mu_{2}-\mu_{1}\right)}{\lambda_{2}\left(\lambda_{1}+\mu_{2}+\beta\right)\left(\mu_{2}-\mu_{1}\right)} & \Longleftrightarrow \\
\lambda_{1} \lambda_{2}\left(\mu_{2}-\mu_{1}\right)+\lambda_{1}^{2}\left(\mu_{2}-\mu_{1}\right) \leq\left(\lambda_{1}+\lambda_{2}+\mu_{2}+\beta\right)\left(\mu_{1}+\beta\right)\left(\mu_{2}+\beta\right) & \Longleftrightarrow \\
\left(\lambda_{1}+\lambda_{2}\right)\left(\mu_{2}-\mu_{1}\right) \lambda_{1} \leq\left(\lambda_{1}+\lambda_{2}+\mu_{2}+\beta\right)\left(\mu_{1}+\beta\right)\left(\mu_{2}+\beta\right) &
\end{aligned}
$$

which is always true by the second part of Condition 2 . Thus $-D_{n}^{\min }(20) \leq D_{n}^{\min }(12)=$ $-D_{n}^{\max }(21)$ and so $v_{n}(. ; 1)$ 's satisfy the monotonicity.

Hence, both statements are true under either Condition 1 or Condition 2. 